\title{
A Hybrid RANS/LES Approach for Predicting Jet Noise
}

\author{
M.E. Goldstein* \\ National Aeronautics and Space Administration, Glenn Research Center, Cleveland, Ohio 44135
}

\begin{abstract}
Hybrid acoustic prediction methods have an important advantage over the current Reynolds averaged Navier-Stokes (RANS) based methods in that they only involve modeling of the relatively universal subscale motion and not the configuration dependent larger scale turbulence. Unfortunately, they are unable to account for the high frequency sound generated by the turbulence in the initial mixing layers. This paper introduces an alternative approach that directly calculates the sound from a hybrid RANS/LES flow model (which can resolve the steep gradients in the initial mixing layers near the nozzle lip) and adopts modeling techniques similar to those used in current RANS based noise prediction methods to determine the unknown sources in the equations for the remaining unresolved components of the sound field. The resulting prediction method would then be intermediate between the current noise prediction codes and previously proposed hybrid noise prediction methods.
\end{abstract}

\section{Introduction}

Current noise prediction methods are usually based on acoustic analogy approaches ${ }^{6,17,5}$ which (as a minimum) require modeling the entire unsteady flow that actually produces the sound and are therefore unlikely to be universal beyond the data base for which they are calibrated. This difficulty would not occur if the sound field were determined from a direct Navier-Stokes (DNS) ${ }^{1}$ solution or even an ordinary Large Eddy Simulation (LES). Unfortunately, the computer resources needed to resolve all the relevant length scales are enormous - often well beyond those available on present day machines. A reasonable compromise would be to use LES simulations with very broad filter widths. This approach was adopted by Bastin, Lafon and Candel ${ }^{12}$, Bodony and Lele ${ }^{7,8,9}$, and others. They found that simulations of this type are reasonably accurate at low frequencies, but significantly underpredict the high frequency component of the spectrum. It is now known that this occurs because these methods do not account for the sound generated by the unresolved turbulence scales, which can correspond to the entire unsteady flow in the thin shear layers near the nozzle lip $^{24}$ as well as to the sub-filter scale motion further downstream ${ }^{9}$. The present paper is an attempt to resolve these difficulties by directly calculating the low frequency sound from a hybrid RANS/LES computation and determining the remaining small scale, or high frequency, sound by modeling the source terms in an appropriate set of linear equations.

The relevant equations are obtained by dividing each of the flow variables in the Navier-Stokes equations into a component that satisfies universal set of conservation laws, which include, among other things, the Reynolds averaged Navier-Stokes (RANS) equations as well as the Favre ${ }^{20}$ filtered Navier-Stokes equations with arbitrary closure models for the sub-filter scale stresses (i.e., the equations that are actually used in most LES calculations) and a residual component that satisfies the Navier-Stokes equations with the base flow equations subtracted out. By introducing new (in general non-linear) dependent variables ${ }^{10,11,12}$ the latter equations can be rewritten in the form of the linearized Euler equations with sources that have the same form as those that would be produced by external stress and heat flux perturbations. The corresponding source strengths, which depend on the non-linear residual Reynolds stresses, can, in principle, be modeled, and the resulting linear equations can be solved by using a Greens' function approach. The result can then be used to obtain an expression for the far field pressure autocovariance in terms of the residual-scale turbulent motion. The latter would only depend on the correlation (i.e., the lower order statistics) of this motion if the base flow were taken to be a steady RANS solution. But the result for any time dependent base flow, such as an LES solution to the filtered Navier-Stokes (or FNS) equations, appears to depend on the detailed instantaneous residual -scale stresses--which are very difficult to model.

Current RANS based noise prediction codes, such as the $\mathrm{JeNo}^{15}$ code, determine the unknown source strengths from experimental data. This cannot be done with prediction methods that involve time dependent base

\footnotetext{
*Senior Scientist, Fellow AIAA
} 
flows because, as a practical matter, experimentalists are limited to documenting the reproducible (i.e., non-random) characteristics of these sources, such as their correlations or other lower order statistics. It is therefore important to derive a formula for the residual sound field that depends only on non-random quantities the can be measured with currently available experimental techniques. The present paper is an attempt to obtain such an equation by exploiting the statistical independence (i.e., the decoupling) of any base flow solution (such as a hybrid RANS/LES simulation) from the detailed sub-scale fluctuations that would occur in an actual experiment. The only coupling that can occur in these simulations is through the base flow stresses and heat flux vector-which are assumed to be modeled in terms of the remaining base flow variables and their derivatives in hybrid RANS/LES computations. The base flow solutions are therefore calculated from a closed set of equations that involve only base flow variables. The chaotic subscale fluctuations in any realization of the flow, i.e., in an actual experiment, can, therefore, not be correlated with the fluctuations in these solutions.

The present paper assumes that the model for the residual (i.e., unresolved) stresses, say $\sigma_{\mu j}^{\prime}$, is constructed from appropriately reduced experimental data (which can, in principle, be obtained from PIV measurements). While the actual (i.e., the experimental) resolved and residual motions are certainly correlated, the experimentally based model for $\sigma_{\mu j}^{\prime}$ must reflect the fact that the experimental residual-scale motions are statistically independent of the fluctuations in the base flow (i.e., the hybrid RANS/LES) solution and must, therefore be uncorrelated with the fluctuations in that solution. This does not, however, mean that the expectation or average value of the residual stresses will be statistically independent of the base flow solution, since the latter is only required to represent a given realization of the flow on a time average basis and can significantly depart from it at any instant of time.

As already indicated, the present paper introduces a hybrid approach in which the base flow corresponds to a typical hybrid RANS/LES solution that goes from a RANS type solution near the nozzle lip (where the initial shear layers are too thin to be resolved by the necessarily coarse LES computation ${ }^{24}$ ) to a (fairly coarse)LES solution further downstream. We adopt the so called universal modeling approach ${ }^{13}$ which assumes that the unknown stresses and heat flux in the base flow equations are modeled by a RANS/LES model that goes from a time average (i.e., RANS type) model near the nozzle lip to a pure spatial LES-type model further downstream. There is no need to complicate matters by requiring that the base flow satisfy the filtered Navier-Stokes equations with inhomogeneous filtering as was done, for example, by Germano ${ }^{4}$ because the combined result (i.e., the base flow plus residual equation solution) is, in principle, exact. But the subscale stress correlations in the residual pressure equation (that go continuously from the full fluctuating stress near the nozzle lip to the instantaneous sub-filter scale stresses further downstream) ultimately have to be modeled.

At high Reynolds numbers the small scale motion is expected to be statistically independent of large energy bearing scales in any realization of the flow. This does not, however, imply that all of the resolved motion will be statistically independent of the subscale motion, because the near cutoff scales are expected to be highly correlated. It is only the hybrid RANS/LES simulation of the resolved scales that is expected to be uncorrelated with the actual, i.e. the experimentally measured, subscale tensor $\sigma_{\mu j}^{\prime}$. But the propagation factor in the Greens' function solution is likely to be dominated by the large energy -bearing scales, and Kolmogorov's ${ }^{3}$ hypothesis (which forms the bases of many of the current subscale turbulence models ${ }^{13}$ ) indicates that the small scale motion should be statistically independent of these scales. It is, therefore, likely that the present decorrelation assumption will be satisfied --at least on a global basis-- in the downstream region of the actual flow (where the implied filter width eventually becomes small relative to the transverse length scale).

The statistical independence assumption is trivially satisfied in the upstream region where the filtered equations reduce to the steady RANS equations because the fluctuating component of the base flow and, therefore, the covariance of the base flow and fluctuating motion, is identically zero. It will not, however, be satisfied in the intermediate blending region. But to the extent that the propagation factor is non-local and the blending region is sufficiently small the present decorrelation assumption should be reasonably well satisfied in any actual realization of the flow.

A major attraction of the current hybrid approach is that the subscale stresses that have to be modeled in the downstream region should be much more universal than the large scale stresses that have to be modeled with the usual RANS based methods. Unfortunately, the present approach still requires full unsteady flow modeling in the initial mixing layers. On the other hand it is now believed that the sound produced by the larger scale motion at the end of the potential core is highly coherent while the sound produced by the small scale motion in the initial mixing layers (as well as by the smaller scale motion at the end of the potential core) is much more random ${ }^{23}$. The latter should therefore be less sensitive to variations in retarded time then the former, which implies that it should also be 
less sensitive to the details of the source structure and, therefore, that relatively universal source models can be constructed.

This is consistent with the prevailing view that RANS based codes can adequately predict the larger angle sound field, which is primarily generated in the initial mixing layers, but tend to under-predict the small angle sound field, which is believed to be generated near the end of the potential core ${ }^{15,23}$. The present approach can be viewed as a framework for directly calculating the latter while retaining the current RANS based approach to calculate the former. But, unlike the RANS approach, the present formalism explicitly accounts for the scattering of the small scale sound by the large scale motion--an effect that was emphasized by Crighton ${ }^{6}$. To the extent that this phenomenon is nonlocal, the present statistical independence assumption should be very appropriate to its computation.

\section{Sub-Filter Scale Equations in Vector Form}

We decompose the pressure $p$, density $\rho$ and velocity $v_{i}, i=1,2,3$, into base flow components $\hat{\rho}, \hat{p}$ and $\tilde{v}_{i}$ and residual components defined by

$$
\rho^{\prime}=\rho-\hat{\rho}, \quad v_{i}^{\prime}=v_{i}-\tilde{v}_{i}, \quad p^{\prime}=p-\hat{p}
$$

where the base flow components satisfy a universal set of conservation laws, which include, among other things, the RANS equations as well as the Favre $^{20}$ filtered Navier-Stokes equations (in which case $\hat{\rho}, \hat{p}$ and $\tilde{v}_{i} \equiv \widehat{\rho v_{i}} / \hat{\rho}$ would correspond to filtered and Favre ${ }^{20}$ filtered quantities respectively ). The residual components can be shown to satisfy the 5 formally linear equations ${ }^{11,12}$

$$
L_{\mu \nu} u_{v}=s_{\mu} \quad \text { for } \quad \mu, v=1,2,3,4,5
$$

where

$$
\left\{u_{v}\right\} \equiv\left\{\rho v_{i}^{\prime}, \pi^{\prime}, \rho^{\prime}\right\} \equiv\left\{\rho v_{1}^{\prime}, \rho v_{2}^{\prime}, \rho v_{3}^{\prime}, \pi^{\prime}, \rho^{\prime}\right\}, \quad \pi^{\prime} \equiv p^{\prime}-\frac{(\gamma-1)}{2} \sigma_{j j}^{\prime}
$$

denotes the solution vector and the first order linear operator $L_{\mu \nu}$ is defined by

$$
L_{\mu v} \equiv \delta_{\mu v} \tilde{D}_{0}+\delta_{v 4} \partial_{\mu}+\partial_{v}\left(\widetilde{c^{2}} \delta_{\mu 4}+\delta_{\mu 5}\right)+K_{\mu v}
$$

with

$$
K_{\mu v} \equiv \partial_{v} \tilde{v}_{\mu}-\frac{1}{\bar{\rho}} \frac{\partial \tilde{\theta}_{\mu j}}{\partial x_{j}} \delta_{v 5}-(\gamma-1)\left(\frac{\partial \tilde{v}_{j}}{\partial x_{j}} \delta_{v 4}-\frac{1}{\bar{\rho}} \frac{\partial \tilde{\theta}_{v j}}{\partial x_{j}}\right) \delta_{\mu 4} \quad i, j=1,2,3
$$

and

$$
\begin{aligned}
& \tilde{D}_{0} \equiv \frac{\partial}{\partial t}+\frac{\partial}{\partial x_{j}} \tilde{v}_{j} ; \quad \tilde{\theta}_{i j} \equiv \delta_{i j} \bar{p}-\tilde{\sigma}_{i j} ; \\
& \partial_{\mu} \equiv \frac{\partial}{\partial x_{j}}, \quad \mu=j=1,2,3 ; \quad \tilde{v}_{\mu}, \tilde{\theta}_{\mu j}, \partial_{\mu}=0, \mu=4,5
\end{aligned}
$$

The source function $S_{\mu}$ is defined by 


$$
s_{\mu} \equiv D_{v j}^{\mu}\left(\sigma_{v j}^{\prime}-\tilde{\sigma}_{v j}\right)-\frac{\gamma-1}{2} D_{i i}^{\mu}\left(\sigma_{j j}^{\prime}-\tilde{\sigma}_{j j}\right)
$$

with

where

$$
D_{v j}^{\mu} \equiv \delta_{v \mu} \frac{\partial}{\partial x_{j}}+\delta_{4 \mu}(\gamma-1) \frac{\partial \tilde{v}_{v}}{\partial x_{j}}
$$

$$
\sigma_{\mu j}^{\prime} \equiv-\rho v_{\mu}^{\prime} v_{j}^{\prime}, \quad v_{4}^{\prime} \equiv-(\gamma-1) h_{0}^{\prime}, \quad v_{5}^{\prime}=0
$$

$\tilde{\sigma}_{5 j}=0$ and $\tilde{\sigma}_{i j}$ and $\tilde{\sigma}_{4 j}$ are the base flow stresses and heat flux vector, which would be given by $\tilde{\sigma}_{i j} \equiv-\hat{\rho}\left(\widetilde{v_{i} v_{j}}-\tilde{v}_{i} \tilde{v}_{j}\right), \tilde{\sigma}_{4 j} \equiv-(\gamma-1)\left[\hat{\rho}\left(\widetilde{h_{0} v_{j}}-\tilde{h}_{0} \tilde{v}_{j}\right)+\frac{1}{2} \tilde{\sigma}_{i i} \tilde{v}_{j}+\tilde{\sigma}_{i j} \tilde{v}_{i}\right]$ if the base flow were chosen to be the FNS equations. Finally, $h_{0} \equiv h+\frac{1}{2} v^{2}, \quad h_{0}^{\prime} \equiv h^{\prime}+\frac{1}{2} v^{\prime 2}$, where $\widetilde{c^{2}}=\gamma R \tilde{T}$ is the squared base flow sound speed, and, as usual, the viscous terms, which are believed to play an insignificant role in the sound generation process, have been neglected. The Latin indices go from 1 to 3 , while the Greek indices go from 1 to 5 .

Equations (2.7) to (2.9) show that the source term in equation (2.2) consists of two parts: a part $\sigma_{i j}^{\prime}$ associated with the residual (or subscale) motion and a base flow component $\tilde{\sigma}_{i j}$ that is the same as the source that appears in the hybrid RANS/LES equations. The subscale motion generated by this latter source component is completely determined by the base flow solution because it is generated by that solution and is determined by equation (2.2) whose coefficients depend on that solution, while the subscale motion generated by $\sigma_{i j}^{\prime}$ is determined by the base flow solution as well as by the experimentally measured residual motion and is, therefore, correlated with both these entities.

Then since equation (2.2) is linear, it makes sense to divide its solution vector into the two components

$$
u_{v}=u_{v}^{s g}+u_{v}^{f}
$$

where

$$
L_{\mu v} u_{v}^{f}=-\left(D_{\lambda j}^{\mu} \tilde{\sigma}_{\lambda j}-\frac{\gamma-1}{2} D_{i i}^{\mu} \tilde{\sigma}_{j j}\right)
$$

and

$$
L_{\mu \nu} u_{v}^{s g}=D_{\lambda j}^{\mu} \sigma_{\lambda j}^{\prime}-\frac{\gamma-1}{2} D_{i i}^{\mu} \sigma_{j j}^{\prime}
$$

It is clear that these results will remain unchanged if $\sigma_{i j}^{\prime}$ and $\tilde{\sigma}_{i j}$ are redefined by

$$
\begin{gathered}
\sigma_{\mu j}^{\prime} \rightarrow-\rho v_{\mu}^{\prime} v_{j}^{\prime}-\bar{\sigma}_{\mu j}(\boldsymbol{x}) \\
\tilde{\sigma}_{\mu j} \rightarrow \tilde{\sigma}_{\mu j}-\bar{\sigma}_{\mu j}(\boldsymbol{x})
\end{gathered}
$$

where $\bar{\sigma}_{\mu j}(\boldsymbol{x})$ is an arbitrary time independent quantity. The overbar will now be used more specifically to denote time averages. Then $\overline{\sigma_{\mu j}^{\prime}(\boldsymbol{y})}$ would equal $\overline{\tilde{\sigma}_{\mu j}(\boldsymbol{y})}$ in the upstream region where the base flow is steady if $\sigma_{\mu j}^{\prime}$ were defined by the original equation (2.8),. Appendix B shows that this will also be approximately true in the downsteam region where the original $\tilde{\sigma}_{\mu j}$ corresponds to the sub-grid stresses and heat flux in the FNS equations and are given 
in the paragraph following equation (2.9). We, therefore, take $\bar{\sigma}_{\mu j}(\boldsymbol{x})$ to be the (approximate) common value of these two quantities and thereby insure that the derived stresses (2.13) and (2.14) have zero time average and, more importantly, that $\tilde{\sigma}_{\mu j} \rightarrow 0$ in the upstream region where the base-flow is steady. The solution to equation (2.10) is, therefore, entirely driven by the initial conditions in this region. Notice that steady sources cannot generate any sound at subsonic Mach numbers.

Since, the coefficients and sources in equation (2.11) are all resolved quantities, i.e. they involve only variables that appear in the base follow equations, it can be solved right along with these equations with no additional modeling and little additional effort. These two sets of equations (i.e., equations (2.11) and the base flow equations) are completely closed once the base flow stress model has been selected. The pressure components of the solution vectors, say $p_{f}^{\prime}$ and $\hat{p}$, can then be added together to obtain the component of the pressure

$$
p_{L} \equiv p_{f}^{\prime}+\hat{p}
$$

that is completely determined by the base flow ( i.e., the hybrid RANS/LES) solution. It may even be possible to calculate these solutions on the same mesh, since $u_{v}^{f}$ is expected to be composed of the larger sub-grid scales ${ }^{7}$. But even if this is not the case, the solution to the linear equation (2.11) should still be relatively inexpensive compared to the base flow solution.

The solution to equation (2.10) can also be used to iteratively calculate at least a portion of the modeled stresses in the hybrid RANS/LES equations in order to gradually introduce the required unsteadiness into the downstream LES solution. This type of calculation, which is sometimes known as mean field theory, should only be required in the blending region between the pure RANS and LES regions. It is expected to provide a better representation of the actual physics than current techniques for inputting unsteadiness into LES computations.

\section{Vector Greens' Function Solution for $\pi_{s g}^{\prime}$}

Solving equations (2.12) is potentially much more difficult than solving equations (2.11), because of the additional source modeling requirement and because its solution can depend upon both the base flow and residual motions. To separate out these effects, we introduce the vector Greens' function ${ }^{21} g_{v \sigma}(\boldsymbol{x}, t \mid \boldsymbol{y}, \tau)$ which satisfies the inhomogeneous linear equations

$$
\left(L_{\mu \nu}\right)_{x, t} g_{v \sigma}(\boldsymbol{x}, t \mid \boldsymbol{y}, \tau)=\delta_{\mu \sigma} \delta(\boldsymbol{x}-\boldsymbol{y}) \delta(t-\tau)
$$

with delta-function type source term together with the causality condition

$$
g_{v \mu}(\boldsymbol{x}, t \mid \boldsymbol{y}, \tau)=0 \quad \text { for } t<\tau
$$

to obtain

$$
\begin{aligned}
u_{v}^{s g}(\boldsymbol{x}, t) & =\int_{V} \int_{-\infty}^{\infty} g_{v \mu}(\boldsymbol{x}, t \mid \boldsymbol{y}, \tau)\left(D_{\lambda j}^{\mu} \sigma_{\lambda j}^{\prime}-\frac{\gamma-1}{2} D_{i i}^{\mu} \sigma_{j j}^{\prime}\right) d \boldsymbol{y} d \tau= \\
& -\int_{V}^{\infty} \int_{-\infty}^{\infty} \tilde{\gamma}_{v j \mu}(\boldsymbol{x}, t \mid \boldsymbol{y}, \tau)\left[\sigma_{\mu j}^{\prime}(\boldsymbol{y}, \tau)-\frac{(\gamma-1)}{2} \delta_{\mu j} \sigma_{k k}^{\prime}(\boldsymbol{y}, \tau)\right] d \boldsymbol{y} d \tau
\end{aligned}
$$

where

$$
\tilde{\gamma}_{v j \mu}(\boldsymbol{x}, t \mid \boldsymbol{y}, \tau) \equiv \frac{\partial g_{v \mu}(\boldsymbol{x}, t \mid \boldsymbol{y}, \tau)}{\partial y_{j}}-(\gamma-1) \frac{\partial \tilde{v}_{\mu}}{\partial y_{j}} g_{v 4}(\boldsymbol{x}, t \mid \boldsymbol{y}, \tau)
$$


From the acoustics perspective the primary interest is in the 4th pressure-like component of (3.3), which only involves the 4 th component vector Greens' function $g_{4 v}(\boldsymbol{x}, t \mid \boldsymbol{y}, \tau)$. The latter quantity can, in principle, be found by solving be solving the system (3.1). But since this consists of 15 first order equations, it turns out to be simpler to compute the adjoint Greens' function $g^{a}{ }^{a} 4(\boldsymbol{y}, \tau \mid \boldsymbol{x}, t)$. Equations (A1) to (A4) show that it is related to $g_{4 v}(\boldsymbol{x}, t \mid \boldsymbol{y}, \tau)$ by the reciprocity relation

$$
g^{a}{ }^{a}(\boldsymbol{y}, \tau \mid \boldsymbol{x}, t)=g_{4 v}(\boldsymbol{x}, t \mid \boldsymbol{y}, \tau)
$$

and satisfies the system

$$
\left(L_{\mu \nu}^{a}\right)_{\mathrm{y}, \tau} g_{v 4}^{a}(\boldsymbol{y}, \tau \mid \boldsymbol{x}, t)=\delta_{\mu 4} \delta(\boldsymbol{x}-\boldsymbol{y}) \delta(t-\tau)
$$

of five first order equations, which are given in component form by (A-5a,b,c). These equations suggest that $\breve{g}_{v 4}(\boldsymbol{y} \mid \boldsymbol{x}: \tau, t-\tau) \equiv g_{\vee 4}^{a}(\boldsymbol{y}, \tau \mid \boldsymbol{x}, t)$ will be a stationary random function of its third argument, $\tau$, which results from the time dependence of the coefficients in $\left(L_{\mu \nu}^{a}\right)_{\mathrm{y}, \tau}$ that are determined from the base flow (i.e., the hybrid RANS/LES) solution and are, therefore, random functions of time. (Notice that this argument would not appear if the base flow were steady.)

It therefore follows from equations (2.3), (3.4) and (3.5) that the 4th component of equation (3.3) can be written (with the obvious notation) as

$$
\pi_{s g}^{\prime}(\boldsymbol{x}, t)=-\int_{V} \int_{-\infty}^{\infty} \gamma_{j \mu}(\boldsymbol{y} \mid \boldsymbol{x}: \tau, t-\tau) \sigma_{\mu j}^{\prime}(\boldsymbol{y}, \tau) d \boldsymbol{y} d \tau
$$

where, like $\breve{g}_{\vee 4}(\boldsymbol{y} \mid \boldsymbol{x}: \tau, t-\tau) \equiv g_{\vee 4}^{a}(\boldsymbol{y}, \tau \mid \boldsymbol{x}, t)$, the propagation factor

$$
\gamma_{j \mu}(\boldsymbol{y} \mid \boldsymbol{x}: \tau, t-\tau) \equiv-\lambda_{j \mu}(\boldsymbol{y} \mid \boldsymbol{x}: \tau, t-\tau)+\frac{(\gamma-1)}{2} \delta_{j \mu} \lambda_{k k}(\boldsymbol{y} \mid \boldsymbol{x}: \tau, t-\tau)
$$

along with

$$
\lambda_{j \mu}(\boldsymbol{y} \mid \boldsymbol{x}: \tau, t-\tau) \equiv\left\{\begin{array}{cc}
\lambda_{j k}(\boldsymbol{y} \mid \boldsymbol{x}: \tau, t-\tau) & \mu=k=1,2,3 \\
\frac{\partial g_{44}^{a}(\boldsymbol{y}, \tau \mid \boldsymbol{x}, t)}{\partial y_{j}} & \mu=4
\end{array}\right.
$$

should be random functions of their third arguments $\tau$, since the third arguments of the six independent components 


$$
\begin{aligned}
& \lambda_{j k}(\boldsymbol{y} \mid \boldsymbol{x}: \tau, t-\tau) \equiv \\
& \quad \frac{1}{2}\left[\frac{\partial g_{4 k}^{a}(\boldsymbol{y}, \tau \mid \boldsymbol{x}, t)}{\partial y_{j}}+\frac{\partial g_{4 j}^{a}(\boldsymbol{y}, \tau \mid \boldsymbol{x}, t)}{\partial y_{k}}\right]-\frac{(\gamma-1)}{2}\left(\frac{\partial \tilde{v}_{k}}{\partial y_{j}}+\frac{\partial \tilde{v}_{j}}{\partial y_{k}}\right) g_{44}^{a}(\boldsymbol{y}, \tau \mid \boldsymbol{x}, t)
\end{aligned}
$$

of $\lambda_{j k}(\boldsymbol{y} \mid \boldsymbol{x}: \tau, t-\tau)$ (which arise from the third argument of $\breve{g}_{v 4}(\boldsymbol{y} \mid \boldsymbol{x}: \tau, t-\tau)$ as well as the $\tau$-dependence of $\left.\partial \tilde{v}_{k} / \partial y_{j}\right)$ also have this property.

\section{Subscale Pressure Autocovariance}

$$
\begin{gathered}
\text { Since } \pi_{s g}^{\prime}(\boldsymbol{x}, t) \rightarrow p_{s g}^{\prime}(\boldsymbol{x}, t) \quad \text { as } x \rightarrow \infty \text {, the far field pressure autocovariance } \\
\overline{\Pi_{s g}(\boldsymbol{x}, \tau)} \equiv \lim _{T \rightarrow \infty} \frac{1}{2 T} \int_{-T}^{T} p_{s g}^{\prime}(\boldsymbol{x}, t) p_{s g}^{\prime}(\boldsymbol{x}, t+\tau) d t \quad \text { for large }|\boldsymbol{x}|
\end{gathered}
$$

becomes

$$
\begin{gathered}
\bar{\Pi}_{s g}=\frac{1}{2 T} \int_{-T}^{T} \int_{-\infty}^{\infty} \iint_{V} \Gamma_{i \sigma j \mu}\left(\boldsymbol{y}, \boldsymbol{\eta} \mid \boldsymbol{x}: t^{\prime}, t^{\prime \prime}, \tau+t^{\prime \prime}-t^{\prime}\right) \sigma_{\sigma i}^{\prime}\left(\boldsymbol{y}, t^{\prime}\right) \sigma_{\mu j}^{\prime}\left(\boldsymbol{y}+\boldsymbol{\eta}, t^{\prime \prime}\right) d \boldsymbol{y} d \boldsymbol{\eta} d t^{\prime} d t^{\prime \prime} \\
=\frac{1}{2 T} \int_{-T}^{T} \int_{-\infty}^{\infty} \iint_{V} \Gamma_{i \sigma j \mu}\left(\boldsymbol{y}, \boldsymbol{\eta} \mid \boldsymbol{x}: t^{\prime}, t^{\prime \prime}+t^{\prime}, \tau+t^{\prime \prime}\right) \sigma_{\sigma i}^{\prime}\left(\boldsymbol{y}, t^{\prime}\right) \sigma_{\mu j}^{\prime}\left(\boldsymbol{y}+\boldsymbol{\eta}, t^{\prime \prime}+t^{\prime}\right) d \boldsymbol{y} d \boldsymbol{\eta} d t^{\prime} d t^{\prime \prime}
\end{gathered}
$$

where

$$
\begin{aligned}
& \Gamma_{j \sigma l \mu}\left(\boldsymbol{y}, \boldsymbol{\eta} \mid \boldsymbol{x}: t^{\prime}, t^{\prime \prime}, \tau+t^{\prime \prime}-t^{\prime}\right) \equiv \int_{-\infty}^{\infty} \gamma_{j \sigma}\left(\boldsymbol{y} \mid \boldsymbol{x}: t^{\prime}, t+\tau-t^{\prime}\right) \gamma_{l \mu}\left(\boldsymbol{y}+\boldsymbol{\eta} \mid \boldsymbol{x}: t^{\prime \prime}, t-t^{\prime \prime}\right) d t \\
& =\int_{-\infty}^{\infty} \gamma_{j \sigma}\left(\boldsymbol{y} \mid \boldsymbol{x}: t^{\prime}, t+\tau+t^{\prime \prime}-t^{\prime}\right) \gamma_{l \mu}\left(\boldsymbol{y}+\boldsymbol{\eta} \mid \boldsymbol{x}: t^{\prime \prime}, t\right) d t
\end{aligned}
$$

is a stationary random function of its fourth and fifth arguments. It follows that

$$
\begin{aligned}
& \Gamma_{i \sigma j \mu}\left(\boldsymbol{y}, \boldsymbol{\eta} \mid \boldsymbol{x}: t^{\prime}, t^{\prime \prime}+t^{\prime}, \tau+t^{\prime \prime}\right) \text { and } \\
& \quad R_{\sigma i \mu j}\left(\boldsymbol{y} ; \boldsymbol{\eta}, t^{\prime \prime}, t^{\prime}\right) \equiv \sigma_{\sigma i}^{\prime}\left(\boldsymbol{y}, t^{\prime}\right) \sigma_{\mu j}^{\prime}\left(\boldsymbol{y}+\boldsymbol{\eta}, t^{\prime \prime}+t^{\prime}\right)
\end{aligned}
$$

are both stationary random functions of $t^{\prime}$.

It is appropriate to regard these two quantities as being uncorrelated in the present approach because the base flow solution, which is determined from a closed set of equations that only involve the base flow variables, should be statistically independent of (i.e., decoupled from) the chaotic subscale motion in the actual experiment used to model these quantities. (In fact the entire experimental flow should eventually become de-correlated from the hybrid LES/RANS simulation where the randomness comes from the numerical round off errors and/or the random initial conditions-- which are themselves uncorrelated with the initial conditions in the experiment ${ }^{18,19}$. It is therefore reasonable to require that any experimentally based model used to represent $\sigma_{\mu j}^{\prime}$ be statistically independent of the base flow motion (and consequently with the coefficients of $L_{\mu v}^{a}$,).

This means that the covariance 


$$
\lim _{T \rightarrow \infty} \frac{1}{2 T} \int_{-T}^{T} \Gamma_{i \sigma j \mu}^{\prime \prime}\left(\boldsymbol{y}, \boldsymbol{\eta} \mid \boldsymbol{x}: t^{\prime}, t^{\prime \prime}+t^{\prime}, \tau+t^{\prime \prime}\right) R_{\sigma i \mu j}^{\prime \prime}\left(\boldsymbol{y} ; \boldsymbol{\eta}, t^{\prime \prime}, t^{\prime}\right) d t^{\prime}
$$

where

$$
\begin{gathered}
\Gamma_{i \sigma j \mu}^{\prime \prime}\left(\boldsymbol{y}, \boldsymbol{\eta} \mid \boldsymbol{x}: t^{\prime}, t^{\prime \prime}+t^{\prime}, \tau+t^{\prime \prime}\right) \equiv \\
\Gamma_{i \sigma j \mu}\left(\boldsymbol{y}, \boldsymbol{\eta} \mid \boldsymbol{x}: t^{\prime}, t^{\prime \prime}+t^{\prime}, \tau+t^{\prime \prime}\right)-\bar{\Gamma}_{i \sigma j \mu}\left(\boldsymbol{y}, \boldsymbol{\eta} \mid \boldsymbol{x}: t^{\prime \prime}, \tau+t^{\prime \prime}\right) \\
R_{\sigma i \mu j}^{\prime \prime}\left(\boldsymbol{y} ; \boldsymbol{\eta}, t^{\prime \prime}, t^{\prime}\right) \equiv R_{\sigma i \mu j}\left(\boldsymbol{y} ; \boldsymbol{\eta}, t^{\prime \prime}, t^{\prime}\right)-\bar{R}_{\sigma i \mu j}\left(\boldsymbol{y} ; \boldsymbol{\eta}, t^{\prime \prime}\right) \\
\bar{\Gamma}_{i \sigma j \mu}\left(\boldsymbol{y}, \boldsymbol{\eta} \mid \boldsymbol{x}: t^{\prime \prime}, \tau+t^{\prime \prime}\right) \equiv \lim _{T \rightarrow \infty} \frac{1}{2 T} \int_{-T}^{T} \Gamma_{i \sigma j \mu}\left(\boldsymbol{y}, \boldsymbol{\eta} \mid \boldsymbol{x}: t^{\prime}, t^{\prime \prime}+t^{\prime}, \tau+t^{\prime \prime}\right) d t^{\prime}
\end{gathered}
$$

and

$$
\bar{R}_{\sigma i \mu j}\left(\boldsymbol{y} ; \boldsymbol{\eta}, t^{\prime \prime}\right) \equiv \lim _{T \rightarrow \infty} \frac{1}{2 T} \int_{-T}^{T} R_{\sigma i \mu j}\left(\boldsymbol{y} ; \boldsymbol{\eta}, t^{\prime \prime}, t^{\prime}\right) d t^{\prime}
$$

should equal zero. It therefore, follows that $\overline{\Pi_{s g}(\boldsymbol{x}, \tau)}$ can be written as an integral

$$
\overline{\Pi_{s g}(\boldsymbol{x}, \tau)}=\int_{-\infty}^{\infty} \iint_{V} \bar{\Gamma}_{i \sigma j \mu}\left(\boldsymbol{y}, \boldsymbol{\eta} \mid \boldsymbol{x}: t^{\prime \prime}, \tau+t^{\prime \prime}\right) \bar{R}_{\sigma i \mu j}\left(\boldsymbol{y} ; \boldsymbol{\eta}, t^{\prime \prime}\right) d \boldsymbol{y} d \boldsymbol{\eta} d t^{\prime \prime}
$$

of the product of two non-random functions.

The first of these functions, which can be expressed as the integral

$$
\bar{\Gamma}_{i \sigma j \mu}\left(\boldsymbol{y}, \boldsymbol{\eta} \mid \boldsymbol{x}: t^{\prime \prime}, \tau+t^{\prime \prime}\right)=\int_{-\infty}^{\infty} \bar{\gamma}_{i \sigma j \mu}\left(\boldsymbol{y}, \boldsymbol{\eta} \mid \boldsymbol{x}: t^{\prime \prime}, t+\tau+t^{\prime \prime}, t\right) d t
$$

over time of the correlation

$$
\bar{\gamma}_{i \sigma j \mu}\left(\boldsymbol{y}, \boldsymbol{\eta} \mid \boldsymbol{x}: t^{\prime \prime}, t+\tau+t^{\prime \prime}, t\right) \equiv \lim _{T \rightarrow \infty} \frac{1}{2 T} \int_{-T}^{T} \gamma_{i \sigma}\left(\boldsymbol{y} \mid \boldsymbol{x}: t^{\prime}, t+\tau+t^{\prime \prime}\right) \gamma_{l \mu}\left(\boldsymbol{y}+\boldsymbol{\eta} \mid \boldsymbol{x}: t^{\prime}+t^{\prime \prime}, t\right) d t^{\prime}
$$

of the two random propagation factors $\gamma_{i \sigma}\left(\boldsymbol{y} \mid \boldsymbol{x}: t^{\prime}, t+\tau+t^{\prime \prime}-t^{\prime}\right)$ and $\gamma_{l \mu}\left(\boldsymbol{y}+\boldsymbol{\eta} \mid \boldsymbol{x}: t^{\prime \prime}, t\right)$, can be thought of as an expected or mean propagation factor. It can be determined as part of the hybrid LES/RANS computation. The second function, which, can be thought of as a source function, describes the low order statistics of the unknown component of the residual fluctuations. It has to be modeled, but it should be much easier to do this than to model the instantaneous values of these fluctuations (which would have to be done if the source and propagation fluctuations were not de-correlated). Notice that this de-correlation only implies that the fluctuation $\Gamma_{i \sigma j \mu}^{\prime \prime}$ (and $\operatorname{not} \bar{\Gamma}_{i \sigma j \mu}$ itself) is independent of the unresolved scales. It may, therefore, be appropriate to paramatize the model for this quantity (i.e., the functional form inferred from the experimental data) and determine the parameters from the hybrid RANS/LES solution. 
We can also think of, $\hat{p}, \tilde{v}_{i}$ and $\hat{\rho}$ and $p^{\prime}, v_{i}^{\prime}$ and $\rho^{\prime}$ as the filtered and unfiltered components of the pressure, velocity and density in the downstream region of anactual flow. Then the adjoint Greens' function, and therefore the propagation factor $\Gamma_{i \sigma j \mu}$, should be dominated by the large energy -bearing scales of that flow, while Kolmogorov's ${ }^{3}$ hypothesis (which forms the bases of many of the current subscale turbulence models ${ }^{13}$ ) indicates that the small scale motion should be statistically independent of these scales. It is, therefore, likely that the present decorrelation assumption will be satisfied --at least on a global basis-- in the downstream region of that flow (where the implied filter width eventually becomes small relative to the transverse length scale).

The statistical independence assumption is trivially satisfied in the upstream region where the base flow equations reduce to the steady RANS equations because the fluctuating component of the base flow and, therefore, the covariance (4.5) is identically zero. It will not, however, be satisfied in the intermediate blending region. But to the extent that the propagation factor is non-local and the blending region is sufficiently small this should have relatively little effect on the predicted sound field.

\section{Conclusions}

An exact equation for the sound generation by the residual turbulence scales in a hybrid RANS/LES based simulation $^{11,12}$ is used to obtain a formula for the residual -scale acoustic pressure autocovariance in terms of the residual -scale turbulence correlation tensor by exploiting the statistical independence of the hybrid RANS/LES solution and the experimentally determined residual motion. The residual acoustic radiation can therefore be calculated by introducing appropriate models for this relatively universal correlation while determining the configuration dependent large scale sound directly from the hybrid RANS/LES solution.

The approach also accounts for the scattering of the small scale sound by the large scale motion-an effect that was emphasized by Crighton ${ }^{6}$. To the extent that this phenomenon is non-local, the present statistical independence assumption should be very appropriate to its computation.

At high Reynolds numbers the small scale motion should be statistically independent of the large energy bearing scales in any realization of the flow. But this does not imply that the resolved scales in the downstream LES region (i.e., the scales larger than the filter width) are all statistically independent of the sub-scale motion, especially near the filter cut off. It is only the hybrid RANS/LES simulation of the filtered scales that is expected to be uncorrelated with the actual, i.e. the experimentally measured, residual-scale tensor $\sigma_{\mu j}^{\prime}$. But to the extent that the propagation factor $\Gamma_{i \sigma j \mu}$ is non-local and dominated by the large energy bearing scales, Kolmogorov's ${ }^{3}$ hypothesis that the small scale motion is statistically independent of these scales suggests that the present de-correlation assumption will be satisfied in any actual realization of the flow. The hope is that the resulting equation (4.10) will accurately predict the subscale pressure autocovariance in that flow to the same extent as the hybrid RANS/LES solution predicts its large scale statistics.

A major attraction of the current hybrid approach is that the sub-scale stresses that have to be modeled in the downstream region should be much more universal than the large scale Reynolds stresses that have to be modeled with the usual RANS based methods. Unfortunately, the present approach still requires full unsteady flow modeling in the initial mixing layers. The hope is that it will be possible to obtain analytically based models for this region that exhibit a fair degree of universality. Other extensions and modifications of this approach are, of course, possible. The base flow can, for example, be taken as a hybrid URANS/LES solution in order to make the near nozzle modeling more universal. It may also be possible to use quasi-linear instability wave models to account for the sound generated in this region ${ }^{14}$.

\section{Appendix A}

The adjoint Greens' function $g^{a}{ }_{v \sigma}(\boldsymbol{y}, \tau \mid \boldsymbol{x}, t)$, which satisfies the adjoint equation ${ }^{21,22,8}$

$$
\left(L_{\mu \nu}^{a}\right)_{\mathrm{y}, \tau} g_{\nu \sigma}^{a}(\boldsymbol{y}, \tau \mid \boldsymbol{x}, t)=\delta_{\mu \sigma} \delta(\boldsymbol{x}-\boldsymbol{y}) \delta(t-\tau)
$$

where 


$$
L_{\mu \nu}^{a} \equiv-\delta_{v \mu} \frac{\tilde{D}}{D \tau}-(\gamma-1) \delta_{\mu 4} \partial_{v}+\left(\frac{\widetilde{c^{2}}}{\gamma-1} \delta_{v 4}+\delta_{v 5}\right) \partial_{\mu}+K_{v \mu}
$$

and

$$
\frac{\tilde{D}}{D \tau} \equiv \frac{\partial}{\partial \tau}+\tilde{v}_{i}(\boldsymbol{y}, \tau) \frac{\partial}{\partial y_{i}}
$$

is related to the direct Greens' function $g_{\sigma v}(\boldsymbol{x}, t \mid \boldsymbol{y}, \tau)$ by the reciprocity relation

$$
g^{a}{ }_{\nu \sigma}(\boldsymbol{y}, \tau \mid \boldsymbol{x}, t)=g_{\sigma v}(\boldsymbol{x}, t \mid \boldsymbol{y}, \tau)
$$

It is determined by the system (3.6) which, when written out in full, becomes ${ }^{8}$

$$
\begin{gathered}
-\frac{\tilde{D} g_{i 4}^{a}}{D \tau}+g_{j 4}^{a} \frac{\partial \tilde{v}_{j}}{\partial y_{i}}+\frac{\widetilde{c^{2}}}{\gamma-1} \frac{\partial g_{44}^{a}}{\partial y_{i}}+\frac{\gamma-1}{\bar{\rho}} \frac{\partial \tilde{\theta}_{i j}}{\partial y_{j}} g_{44}^{a}+\frac{\partial g_{54}^{a}}{\partial y_{i}}=0 \\
-\frac{\tilde{D} g_{44}^{a}}{D \tau}-(\gamma-1)\left(\frac{\partial g_{i 4}^{a}}{\partial y_{i}}+g_{44}^{a} \frac{\partial \tilde{v}_{j}}{\partial y_{j}}\right)=\delta(\boldsymbol{x}-\boldsymbol{y}) \delta(t-\tau) \\
-\frac{\tilde{D} g_{54}^{a}}{D \tau}-\frac{1}{\bar{\rho}} \frac{\partial \tilde{\theta}_{i j}}{\partial y_{j}} g_{i 4}^{a}=0
\end{gathered}
$$

Then when $\boldsymbol{x}, \boldsymbol{y} \rightarrow \infty, \widetilde{c^{2}} \rightarrow c_{0}^{2}=$ constant and

$$
\begin{aligned}
& -\frac{\partial g_{i 4}^{a}}{\partial \tau}+\frac{c_{0}^{2}}{\gamma-1} \frac{\partial g_{44}^{a}}{\partial y_{i}}=0 \\
& -\frac{\partial g_{44}^{a}}{\partial \tau}-(\gamma-1) \frac{\partial g_{i 4}^{a}}{\partial y_{i}}=\delta(\boldsymbol{x}-\boldsymbol{y}) \delta(t-\tau) \\
& \frac{\partial g_{54}^{a}}{\partial \tau}=0
\end{aligned}
$$

which shows that $g_{44}^{a}$ satisfies the inhomogeneous wave equation

$$
-\frac{\partial^{2} g_{44}^{a}}{\partial \tau^{2}}+c_{0}^{2} \frac{\partial^{2} g_{44}^{a}}{\partial y_{i} \partial y_{i}}=\delta(\boldsymbol{x}-\boldsymbol{y}) \frac{\partial}{\partial \tau} \delta(t-\tau)
$$

The relevant solution which satisfies the causality condition 


$$
g_{44}^{a}(\boldsymbol{y}, \tau \mid \boldsymbol{x}, t)=0 \text { for } \quad t<\tau
$$

is

$$
g_{44}^{a}(\boldsymbol{y}, \tau \mid \boldsymbol{x}, t)=\frac{1}{4 \pi|\boldsymbol{x}-\boldsymbol{y}| c_{0}^{2}} \frac{\partial}{\partial \tau} \delta\left(\tau-t+\frac{|\boldsymbol{x}-\boldsymbol{y}|}{c_{0}}\right)
$$

So that when $x \equiv|\boldsymbol{x}| \rightarrow \infty$

$$
g_{44}^{a} \rightarrow \frac{1}{4 \pi x c_{0}^{2}} \frac{\partial}{\partial \tau} \delta\left(\tau-\frac{\boldsymbol{x} \cdot \boldsymbol{y}}{x c_{0}}-t+\frac{x}{c_{0}}\right)
$$

Notice that the far field adjoint Greens' function can be calculated directly by allowing $x \equiv|\boldsymbol{x}| \rightarrow \infty$ in (A7).

\section{Appendix B}

Since the subscale motion in the downstream region is likely to behave incompressibly ${ }^{13}$, we neglect density variations in this appendix and set $\tilde{\bullet}=\hat{\bullet}$. Since the subscale stress model is specified independently of the choice of filter in most LES computations, we are at liberty to choose the implied filter more or less arbitrarily and can even choose it to be a general space-time filter ${ }^{13}$. We therefore define it by ${ }^{4}$

$$
\hat{f} \equiv \bar{f}+\langle f\rangle-\overline{\langle f\rangle}=\bar{f}+\left\langle f^{\prime \prime}\right\rangle
$$

where $\langle\bullet\rangle$ denotes an arbitrary spatial filter, as before, $\bullet$ - denotes a time average and $f^{\prime \prime} \equiv f-\bar{f}$ denotes the fluctuating component of $f$. Recall that $\sum_{n} a_{n}\langle\bullet\rangle_{n}$ will be a filter when the $\langle\bullet\rangle_{n}$ are filters and the constants $a_{n}$ sum to unity, that the filter of a filter is a filter and that the time average commutes with spatial filtering. It follows that

$$
\overline{\hat{f}}=\bar{f}
$$

and, therefore, in view of equations (2.9) and the following paragraph, that

$$
\begin{aligned}
\overline{\sigma_{i j}^{\prime}(\boldsymbol{y})}-\overline{\tilde{\sigma}_{i j}(\boldsymbol{y})}=-\rho\left(\overline{v_{i}^{\prime} v_{j}^{\prime}}-\overline{v_{i} v_{j}}+\overline{\hat{v}_{i} \hat{v}_{j}}\right)=\rho\left(\overline{\hat{v}_{i} v_{j}^{\prime}}+\overline{\hat{v}_{i} v_{j}^{\prime}}\right) \\
=\rho\left[\overline{\left(\hat{v}_{i}-\overline{\hat{v}}_{i}\right)\left(\bar{v}_{j}^{\prime}-\vec{v}_{j}^{\prime}\right)+\left(\hat{v}_{j}-\overline{\hat{v}}_{j}\right)\left(v_{i}^{\prime}-\vec{v}_{i}\right)}\right]
\end{aligned}
$$

which is just the sum of the covariances of $\hat{v}_{i}$ with $v_{j}^{\prime}$ and of $\hat{v}_{j}$ with $v_{i}^{\prime}$ and should therefore vanish when these quantities are uncorrelated.

Now as explained above, they cannot be completely uncorrelated in any actual realization of the downstream flow because the filtered and sub-filter scales will be strongly correlated near the filter cutoff. But, as 
argued by Kolmogorov ${ }^{3}$, the small scale motion should be de-correlated from the large energy containing scales at high Reynolds numbers. We, therefore, expect that

$$
\overline{\sigma_{i j}^{\prime}(y)} \approx \overline{\tilde{\sigma}_{i j}(y)}
$$

when the cutoff for the filter $\langle\bullet\rangle$ is within the inertial sub-range. The result should be exact in the present context where the subscale motion is determined from an actual realization of the flow--and therefore uncorrelated with $\hat{v}_{i}$. Equation (B-4) should also hold for $\overline{\sigma_{4 j}^{\prime}(\boldsymbol{y})}$ and $\overline{\tilde{\sigma}_{4 j}(\boldsymbol{y})}$ to the extent that $v^{2}$ is small compared to $h$.

We can therefore choose the undefined function of position in equations (2.13) and (2.14) to be the common value of these two quantities and thereby obtain

$$
\overline{\sigma_{\mu j}^{\prime}(y)} \approx \overline{\tilde{\sigma}_{\mu j}(y)} \approx 0
$$

when $\sigma_{\mu j}^{\prime}$ and $\tilde{\sigma}_{\mu j}$ are defined in this fashion. This means that they are pure fluctuating quantities, which is an appropriate requirement to impose on a true sound source.

\section{References}

${ }^{1}$ Freund, J.B. Noise sources in a low-Reynolds-number turbulent jet at Mach 0.9, J. Fluid Mech., vol. 438, 277-305. (2001)

${ }^{2}$ Bastin, F., Lafon, P., and Candel, S. Computation of Jet Mixing Noise due to Coherent Structures: The Plane Jet Case, J. Fluid Mech., vol. 335, pp. 261-304. (1997)

${ }^{3}$ Kolmogorov, A.N., A refinement of previous hypotheses concerning the local structure of turbulence in a viscous incompressible fluid at high Reynolds number, J. Fluid Mech. Vol.13, pp. 82-85, 1962)

${ }^{4}$ Germano, M., Properties of the RANS/LES filter Theo. And Comp. Fluid Dynamics, vol. 7, pp. 225-231, 2004

${ }^{5}$ Lilley, G.M., "The Radiated Noise from Isotropic Turbulence with Applications to the Theory of Jet Noise," Journal of Sound Vibration, vol. 190pp. 463-476 (1996)

${ }^{6}$ Crighton,D.G. Why Do the Acoustics and the Dynamics of a Hypothetical Mean Flow Bear on the Issue of Sound generated by Turbulence? In Mechanics of Sound Generation in Flows (E.-A, Muller, ed.) Springer-Verlag, Heidelberg (1979)

${ }^{7}$ Bodony, D.J. and Lele, S.K. Spatial scale decomposition of shear layer turbulence and the sound sources associated with the missing scales in a large-eddy simulation, AIAA Paper No. 2002-2454, $8^{\text {th }}$ AIAA/CEAS Aeroacoustics Conference, Breckenridge, CO. (2002)

${ }^{8}$ Bodony, D.J. and Lele, S.K., A Stochastic Subgrid Scale Model for Noise Predictions of Subsonic Jets, AIAA Paper No. 2003-3252, $9^{\text {th }}$ AIAA/CEAS Aeroacoustics Conference, Hilton Head, South Carolina. (2003)

${ }^{9}$ Bodony, D. J. and Lele, S. K., On using large-eddy simulation for the prediction of noise from cold and heated turbulent jets, Phys. Fluids 17, 085103 (20 pages) (2005)

${ }^{10}$ Goldstein, M.E. Some recent developments in jet noise modeling, Program and Abstracts of the $6{ }^{\text {th }}$ International Congress on Sound and Vibration, Copenhagen, Denmark, p. 21. (1999)

${ }^{11}$ Goldstein, M.E., A unified approach to some recent developments in jet noise theory. International Journal of Aeroacoustics, vol. 1, no. 1, pp. 1-16. (2002)

${ }^{12}$ Goldstein, M.E., “A Generalized Acoustic Analogy,” Journal of Fluid Mechanics, vol. 488, pp. 315-333. (2003) 

2002

${ }^{13}$ Sagaut, P., Large Eddy Simulation for Incompressible Flows, Springer, Berlin, p.12, p 75 and ff., p.320 and pp. 323-324,

${ }^{14}$ Mankbadi, R.R. and Liu, J.T.C. Sound generated aerodynamically revisited: Large scale structure in a turbulent jet as a source of sound, Phil. Trans. Roy. Soc. London, A vol 298, pp183-217, (1984)

${ }^{15}$ Khavaran, A. and Bridges, J., Modeling of Turbulence Generated Noise in Jets, AIAA paper \# 2004-2983, Presented at the $10^{\text {th }}$ AIAA/CEAS Aeroacoustics Conference \& Exhibit, Manchester, U. K. (2004)

${ }^{16}$ Lighthill, M.J. On Sound Generated Aerodynamically: I. General Theory, Proc. R. Soc. Lond., A211, pp. 564-587. (1952)

${ }^{17}$ Lighthill, M.J. On sound Generated Aerodynamically: II. Turbulence as a source of sound, Proc. R. Soc. Lond., A222, pp. 1-32. (1954)

${ }^{18}$ Lesier, M., , Turbulence in Fluids, Martinus Nijhoff Publishers, Dordrecht , (1987) p.230

${ }^{19}$ Pope, S.B., Turbulent Flows, Cambridge University Press, (2000) pp.612-613 and 229-230

${ }^{20}$ Favre, A., Statistical equations of turbulent gases, In Problems of Hydrodynamics and Continuum Mechanic, SIAM, Philadelphia. (1969)

${ }^{21}$ Morse, P.M. and Feshbach, H. ,Methods of theoretical physics, McGraw-Hill. (1953)

${ }^{22}$ Tam, C.K.W. \& Auriault, L. Mean Flow Refraction Effects on Sound Radiated from Localized Sources in a Jet. J. Fluid Mech. 370 pp. 149-174 (1998)

${ }^{23}$ Tam, C K.W.; Pastouchenko, N. N. , Fine-Scale Turbulence Noise from Dual-Stream Jets, AIAA Journal vol. 44 no. 1 , pp.90-101(2006)

${ }^{24}$ DeBonis, J. R., Progress Towards Large-Eddy Simulations for Prediction of Realistic Nozzle Systems, AIAA Paper No. 2006-487, 44 $4^{\text {th }}$ AIAA Aerospace Sciences Meeting and Exhibit, Reno, Nevada. (2003) 